\title{
EchoGéo
}

$10 \mid 2009$

La piraterie

\section{Lutte contre la piraterie : faut-il faire appel aux sociétés de sécurité privées?}

Témoignages croisés

(2) OpenEdition

Journals

Édition électronique

URL : https://journals.openedition.org/echogeo/11437

DOI : $10.4000 /$ echogeo. 11437

ISSN : 1963-1197

Éditeur

Pôle de recherche pour l'organisation et la diffusion de l'information géographique (CNRS UMR 8586)

\section{Référence électronique}

"Lutte contre la piraterie : faut-il faire appel aux sociétés de sécurité privées ? », EchoGéo [En ligne],

10 | 2009, mis en ligne le 14 octobre 2009, consulté le 16 septembre 2021. URL : http://

journals.openedition.org/echogeo/11437; DOI : https://doi.org/10.4000/echogeo.11437

Ce document a été généré automatiquement le 16 septembre 2021.

EchoGéo est mis à disposition selon les termes de la licence Creative Commons Attribution - Pas d'Utilisation Commerciale - Pas de Modification 4.0 International (CC BY-NC-ND) 


\title{
Lutte contre la piraterie : faut-il faire appel aux sociétés de sécurité privées?
}

\author{
Témoignages croisés
}

\section{Le point de vue d'un armateur français ${ }^{1}$}

2 Eric Frécon: Quel est l'environnement juridique dans lequel opèrent les armateurs soucieux de protéger leur navire avec des hommes en armes?

3 Sur un navire, dans les eaux nationales d'un pays, la protection armée s'effectue dans le cadre du droit maritime dudit pays. Ces eaux nationales s'entendent sans contestation aux douze nautiques mais dans certains cas, comme aux Etats-Unis, elles sont prolongées jusqu'à la limite de la ZEE soit 200 milles. Ainsi, dans le détroit de Malacca, l'usage de ces sociétés est contraint par les droits de la Malaisie et de l'Indonésie. Comme ces droits sont relativement peu protecteurs concernant les droits de l'homme, la latitude d'action des ces sociétés est grande (légitime défense armée, etc.) et la responsabilité potentielle de l'armateur les employant est très faible.

4 Il en va tout autrement du golfe d'Aden. Parce que la Somalie n'exerce objectivement plus sa souveraineté sur mer dans les douze milles et parce que ni la Somalie ni le Yémen n'estime que leur souveraineté s'étend à leur ZEE hors exploitation des ressources sous-marines, l'embarquement et l'usage de sociétés de protection privées s'y effectue selon le droit du pavillon du navire concerné. Et là, cela se complique ! Par exemple, la France dispose d'une jurisprudence beaucoup moins « permissive » que les Etats-Unis concernant l'usage de la force en légitime défense.

5 - Que se passe-t-il précisément en cas d'échanges de tirs?

6 Je prends un exemple récent : un navire-mère a été attaqué en février dernier par un navire de la marine indienne. Il a été coulé avec les pirates à bord mais aussi, malheureusement, avec son équipage original. En effet, les navires mères sont souvent la première prise d'un groupe de pirates (chalutier, petit cargo ou dow, etc.). Les pirates gardent l'équipage à bord comme bouclier humain. Si une société privée, placée sur un navire marchand français, tue un otage d'un pirate ou même un pirate sans que nous 
puissions ensuite prouver qu'il s'agissait vraiment d'un pirate, il est tout à fait envisageable que l'armateur employeur de cette société soit poursuivi pour homicide volontaire avec préméditation. Le fait d'employer des hommes en armes suffit à caractériser la préméditation puisque nul ne peut ignorer que les armes pourront être utilisées. Pire encore, si au cours de l'échange de tirs avec les pirates un homme d'équipage marchand français est blessé, l'armateur sera accusé d'avoir, par son action de location d'une société privée, contribué à l'escalade ayant mené au décès du marin. La responsabilité de l'armateur sera clairement engagée.

8 Il est intéressant de noter que certains avocats et ONG se sont fait un devoir de défendre les pirates, souvent très efficacement étant donné l'absence de règles claires sur le «droit de suite » qu'ont les marines occidentales dans les eaux somalies : par exemple Lawyers of the World dont une figure de proue est maitre Avi Singh. Il est très impliqué dans la défense des droits des présumés pirates qui sont souvent jugés au Kenya en vertu d'un accord passé avec les puissances occidentales.

9 Le cas des pirates emprisonnés en France suite au détournement du Ponant est très complexe : il n'est pas certain du tout qu'ils soient condamnés in fine, leur arrestation pouvant aisément être considérée comme arbitraire ou illégale.

10 Tout cela couplé au fait que les sociétés privées ont souvent un passé sulfureux nous a amené à ne jamais faire appel à elles et à privilégier pour les navires les plus fragiles la mise à bord de commandos de la Marine Nationale.

11 Le point de vue de David Hornus, directeur de Risksgroup ${ }^{2}$

12 Eric Frécon : La communauté internationale et les Etats apportent-ils une réponse adaptée et efficace au problème de la piraterie?

13 L'efficacité de l'opération Atalante peut-être est sujette à caution. Tout d'abord, elle agit sur les conséquences et non sur les causes de la piraterie; ensuite l'étendue maritime à surveiller est tellement vaste et la densité du trafic telle que l'opération ne peut pas protéger tous les navires; enfin, les forces navales sont limitées par les règles d'engagement et les restrictions juridiques. Si l'opération Atalante a eu des effets localisés sur l'action des pirates, ceux-ci, loin d'avoir été impressionnés par la présence d'une telle armada, ont tout simplement augmenté leur rayon d'action. Ils opèrent désormais jusqu'à 500 milles nautiques de leurs bases de la côte est de la Somalie et à 175 milles nautiques des Seychelles.

14 Selon un rapport de l'Institut canadien de la défense et des affaires étrangères ${ }^{3}$ : « il est vain, au regard du coût, de croire qu'une flotte navale et aérienne de l'ampleur sans précédent de celle actuellement déployée, puisse être maintenu sur le long terme ». Le coût de l'opération pour l'EU NAVFOR s'élèverait à 300 millions de dollars pour 2008, somme payée par les contribuables européens.

15 La Marine nationale, parallèlement à sa participation à l'opération Atalante, propose de mettre des militaires armés (fusiliers marins, commandos marine) à bord des navires privés français qui en font la demande.

16 Il est d'ailleurs paradoxal de constater que les armateurs ou corporations d'armateurs français pusillanimes qui répètent à satiété que la présence de personnels armés à bord des navires mènera forcement à une escalade de la violence sont les premiers à demander à bénéficier de cette assistance armée de la Marine Nationale. 
17 Si le surcoût occasionné par cette " prestation » est facturé aux bénéficiaires, c'est bien le contribuable français qui en supporte, indirectement, le coût. Ce type d'arrangement, dont nul ne connaît les conditions tarifaires ${ }^{4}$, s'apparente de facto à du mercenariat étatique voire à une privatisation de l'armée. desquels des rançons conséquentes seront réclamées. D'écumeur des mers, il devient " ravisseur des mers ». Ainsi, à défaut d'avoir souscrit une assurance "Kidnapping et rançon", ce sont les armateurs et les Etats dont sont originaires les membres d'équipages qui supportent les coûts liés au piratage d'un navire. Notons que cette assurance impose généralement des formations aux équipages ainsi qu'une présence à bord, pas toujours armée, même si selon nous cela ne sert à rien et met en danger la vie des agents embarqués. 
24 En cas d'intervention régalienne, le flou le plus opaque est entretenu concernant le paiement de la rançon et surtout l'origine des fonds.

Il convient donc désormais que chaque équipage puisse bénéficier des formations en amont et des mesures de protections adéquates à bord. Les armateurs doivent être correctement assurés et doivent pouvoir bénéficier de l'assistance ad hoc de prestataires privés.

Or les armateurs français entretiennent une défiance irrationnelle à leur encontre allant même, pour certains, jusqu'à affirmer que les SSP ont souvent un passé sulfureux! En réponse et en ce qui nous concerne, notre société est response team member exclusif de Corporate Risk International ${ }^{8}$, société américaine composée majoritairement d'anciens hauts dirigeants du FBI. Cette société est à l'origine d'une joint venture avec Veritas International, dont le directeur n'est autre que le Vice-Amiral Scott Liedbetter, de la Royal Navy.

\section{NOTES}

1. Parce qu'encore en exercice, l'armateur a choisi de garder l'anonymat.

2. PHAM, J. Peter, 30/4/2009. "Countering Somali piracy by involving the private sector". World Defense Review. http://worlddefensereview.com/pham043009.shtml (consulté le 24/9/2009). 3. LENOX, Patrick, December 2008. Contemporary Piracy off the Horn of Africa. Calgary: Canadian Defense and Foreign Affairs Institute, 24 p. http://www.qaranimo.com/2009/feb/ Contemporary_Piracy_off_the_Horn_of_Africa.pdf (consulté le 24/9/2009)

4. Le ministère de la défense belge avait proposé que des soldats belges soient mis à bord des navires. Le prix évoqué à l'époque était de $115000 €$ pour huit personnels armés pour une durée de huit jours. Soit environ le même coût que les prestataires privés.

5. Le Monde, $24 / 9 / 2008$

6. Al Jazeera, 9/9/2009

7. PLOCH, Lauren et al., 20/4/2009. Piracy off the Horn of Africa, CRS Report for Congress, 28 p. http://www.nytimes.com/packages/pdf/topics/piracycrs.pdf (consulté le 24/9/2009)

8. Corporate Risk International est sous mandat exclusif de Professional Indemnity Agency, Inc. (PIA), agence de souscription de la Lloyd's et filiale de HCC Insurance Holdings Inc.

INDEX

Thèmes : Sur le Champ - Sur le Terrain 